\title{
Predicting the Effect of Parathyroidectomy on Patient Survival in Secondary Hyperparathyroidism with Machine Learning
}

\author{
Oktoria and Cheng-Hong Yang \\ Department of Electronic Engineering, National Kaohsiung University of Applied Sciences, Kaohsiung, Taiwan \\ Email: oktoria.ok@gmail.com, chyang@cc.kuas.edu.tw \\ Jin-Bor Chen \\ Division of Nephrology, Department of Internal Medicine, Kaohsiung Chang Gung Memorial Hospital and Chang \\ Gung University Collages of Medicine, Kaohsiung, Taiwan \\ Email: chenjb1019@gmail.com
}

\begin{abstract}
The main goal of parathyroidectomy (PTX) is to remove the offending gland(s) while protecting the remaining normal parathyroid glands as well as the recurrent laryngeal nerves and the thyroid gland. In this study, the writer hypothesized that Machine Learning (ML) could predict the effect of PTX based on readily available clinical and laboratory indicators. There were 158 consecutive HD patients who underwent PTX before 2009 and 275 consecutive hemodialysis (HD) patients without PTX as controls from those visiting the Kaohsiung Chang Gung Memorial Hospital, Taiwan between 2009 and 2013. The study first held by testing several categories of supervised ML classifiers: 1) Bayesian network classifier, 2) k-Nearest Neighbors, 3) rule-based classifiers, and 4) treebased classifiers. All ML classifiers were tested using 10-fold cross validation. The performance of each classifier was evaluated based on sensitivity (recall), specificity, positive predictive value (precision), area under the Receiver Operating Characteristic (ROC) curve, and overall accuracy. After testing $>20$ different algorithms, we selected tree-based classifier (Random Forest) that has the highest value of correctly classified instances, namely $76.91 \%$ (area under receiver operating characteristic $=0.78$ ).
\end{abstract}

Index Terms-parathyroidectomy, machine learning, classifier, random forest

\section{INTRODUCTION}

Secondary hyperparathyroidism (HPT) means the parathyroid glands are overproducing parathyroid hormone in an attempt to help body increase the amount of calcium in the blood. It is the only job of the parathyroid glands to maintain calcium levels and the four parathyroid glands will increase the production of their hormone (parathyroid hormone PTH) if the calcium is too low [1]. Secondary HPT occurs most commonly "secondary" to chronic renal failure (CRF). For this reason, secondary HPT is frequently referred to as renal HPT [2]. Secondary HPT can be treated by PTX

Manuscript received January 4, 2017; revised April 27, 2017. operation which can remove one or more of the parathyroid glands.

Parathyroidectomy is necessary when calcium levels are elevated, if there is a complication of HPT (such as kidney stone, osteoporosis, and bone fractures), or if a patient is relatively young. The main goal of PTX is to remove the offending gland(s) while protecting the remaining normal parathyroid glands as well as the recurrent laryngeal nerves and the thyroid gland [3]. The effect of PTX can be predicted with Machine Learning (ML). There are many possibilities for how ML can be used in healthcare, and all of them depend on having sufficient data and permission to use it [4]. An example of healthcare providers can take advantage of ML is being able to classify the quality of Electrocardiogram (ECG) signal. Tanatong [5] proposed an approach for signal quality classification in continuous cardiac monitoring using wireless sensors. ECG signal recordings captured while subjects are performing Activities of Daily Living (ADLs). Using a $k$-Nearest Neighbor $(k N N)$ algorithm and statistical features, 5-second ECG segments were classified into good-quality and badquality levels.

Machine Learning (ML) aims at providing computational methods for accumulating, changing and updating knowledge in intelligent systems, and in particular learning mechanism that will help us to induce knowledge from examples or data. ML provides methods, techniques, and tools that can help solving diagnostic and prognosis problems in a variety of medical domains. It is argued that the successful implementation of ML methods can help the integration of computer-based systems in the healthcare environment providing opportunities to facilitate and enhance the work of medical experts and ultimately to improve the efficiency and quality of medical care [6]. In this study, it is hypothesized that ML could predict the effect of PTX based on readily available clinical and laboratory indicators. 


\section{METHODS}

Scheduling regular treatments are applied to outpatient hemodialysis (HD) who attended the Kaohsiung Chang Gung Memorial Hospital, Taiwan. There were 665 patients who got treatment 3 times a week. Their laboratory blood values were recorded from January 1, 2009 to December 31, 2013. Based on the exclusion criteria reported in [7], the invalid HD patients were (1) patients who had initiated regular HD after January 1, 2009; (2) patients who were older than 90 years; (3) patients who received PTX after January 1, 2009; (4) patients transferred to other medical facilities; and (5) patients whose information was incomplete and/or those who were lost to follow-up during the study period. After eliminating those which fell out because of the exclusion criteria, the final sample was 433 patients. There were 158 consecutive HD patients who underwent PTX before 2009 and 275 consecutive hemodialysis (HD) patients without PTX as controls.

Machine Learning (ML) is a collection of different methods or algorithms, each with differing mechanisms of classification [8]. An algorithm can model a problem from an input data. Input data is called training data and has known label or result such as patients who underwent PTX and patients without PTX. A model is prepared through a training process in which it is required to make predictions and is corrected when those predictions are wrong. The training process continues until the model achieves a desired level of accuracy on the training data. In order to accomplish the task, the ML software platform available is used from the Waikato Environment for Knowledge Analysis (version 3.7.12, Weka). The Weka software suite contains a library of algorithms that build predictive models by learning from examples provided in supplied datasets. Each algorithm was trained using our patient dataset, which included 20 attributes. A 5-year average value was calculated for each blood analysis variable. Ten blood analysis variables were considered as continuous variables $(\mathrm{Hb}$, albumin, blood urea nitrogen, creatinine $[\mathrm{Cr}]$, potassium $[\mathrm{K}]$, corrected serum calcium [Ca], phosphate [P] levels, intact-parathyroid hormone [iPTH] levels, ferritin levels, and $\mathrm{Kt} / \mathrm{V})$, whereas the remaining variables (URR, CT ratio) were considered categorical variables. URR was categorized as either less than $65 \%$ or $\geq 65 \%$, whereas the CT ratio was categorized as either less than $50 \%$ or $\geq 50 \%$.

The study began by testing several categories of supervised ML classifier : 1) Bayesian network classifier, 2) $k$-Nearest Neighbors, 3) rule-based classifiers, and 4) tree-based classifiers. After testing >20 different algorithms, we selected tree-based (Random Forest) for additional study as it maximized accuracy and transparency. Random Forest is one of the most popular and most powerful machine learning algorithms. It is a type of ensemble machine learning algorithm called Bootstrap Aggregation or bagging. Random forest changes the algorithm for the way that the sub-trees are learned so that the resulting predictions from all of the subtrees have less correlation.
All ML classifiers were tested using 10-fold cross validation. The performance of each classifier was evaluated based on sensitivity (recall), specificity, positive predictive value (precision), area under the Receiver Operating Characteristic (ROC) curve, and overall accuracy. Continuous variables were estimated by independent 2-sample t-test. The effects of PTX on clinical variables were tested by multivariate linear regression, after adjusting for age, gender, diabetes mellitus, and hemodialysis duration. Categorical variables were statistically compared using chi-square test. The 95 $\%$ confidence interval $(95 \% \mathrm{CI})$ and a $P$ value were used to determine statistical significance. A $P$ value less than 0.05 was considered statistically significance.

\section{RESULT}

The baseline characteristics of the study participants are presented in Table I. A total of 158 PTX patients with 14.54 years mean HD duration and 275 controls (without PTX) with 9.85 years mean HD duration were enrolled between 2009 and 2013. The PTX patients showed significant differences in HD duration, age groups, diabetes mellitus, iPTH levels, Hct, BUN, Cr, and $\mathrm{Ca}$ compared with those without PTX. For PTX patients, BUN, Cr, and Hct were higher, whereas $\mathrm{Ca}$ and $\mathrm{iPTH}$ levels were lower than those without PTX.

PTX patients had a much lesser mean calcium and iPTH levels $(9.11 \pm 0.0085 \mathrm{mg} / \mathrm{dL}$ and $221.32 \pm 3.44$ $\mathrm{pg} / \mathrm{mL}$, respectively) compared with that of controls $(9.40$ $\pm 0.0077 \mathrm{mg} / \mathrm{dL}$ and $430.11 \pm 5.29 \mathrm{pg} / \mathrm{mL}$, respectively). Table II summarizes the effect of PTX on clinical variables adjusted for age, sex, diabetes mellitus, and HD duration. It showed significant differences in $\mathrm{Hb}, \mathrm{BUN}$, $\mathrm{Cr}$, Hct, $\mathrm{Ca}$ and iPTH levels between PTX patients and controls.

After testing a variety of ML methods, it is found that a Random forest predicted the effect of parathyroidectomy more accurate. A graphic representation of the network structure is shown in Fig. 1. The Random forest resulted in a test set accuracy of $78.98 \%$ and area under the ROC curve of 0.786 (Table III).

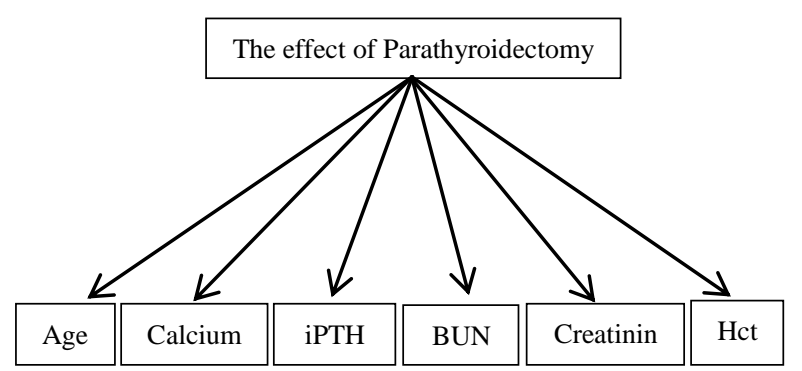

Figure 1. Graphic structure of the random forest classifier. Each arrow represents a conditionally dependent relationship.

\section{DISCUSSION}

During a 5-year cohort study, it is found that PTX patients had higher level of $\mathrm{Hb}, \mathrm{BUN}, \mathrm{Cr}$, and Hct, as well as lower blood $\mathrm{Ca}$ and iPTH levels, compared with those who did not undergo PTX. The findings of the 
present study provide further evidence for the benefits of PTX in HD patients. For patients on dialysis, levels of iPTH that are within or below the normal range of the assay area generally indicative of low bone turnover and levels of iPTH that are greater than 2-3 times the upper normal range of the assay are generally indicative of high bone turnover [9]. No change was detected in fracture rates up to 1 year after parathyroidectomy, given that fractures occurred infrequently [10].

Both high calcium and phosphate are implicated in contributing to an increased cardiovascular calcification in patients on renal replacement therapy (RRT) and an increased cardiovascular and all-cause mortality. PTX was associated with improved survival in patients on maintenance dialysis but not in patients with renal allograft. Moreover, PTX improves calcium and phosphate balance [11].

Several studies have found that parathyroidectomy is underutilized [12], and the undertreatment seems to hinge on at least 2 factors : first, a lack of diagnostic awareness, and second, deferment of evaluation [13], [14]. The informatics tools reported here can prompt timely referral for further evaluation and treatment. Importantly, this tool utilizes readily available clinical data and provides automated prediction without additional cost or invasive testing. Our Random forest to predict the effect of PTX could become even more accurate as it is "trained" on more patients. Although the present study provided promising evidence for PTX in HD patients, it presents some limitations. First, the study was conducted in just one HD unit. The surgeon's skill, preoperative assessment, and quality of care by nephrologists will affect the outcomes of PTX in HD patients. Second, the determination of test-set accuracies by cross-validation within the dataset, thus making it difficult to predict how the algorithm would perform a different population. Finally, future analysis of the tool should include laboratory values taken at multiple time points to reflect actual clinical practice which may potentially increase the accuracy for the most challenging cases.

TABLE I. BASELINE CHARACTERISTIC OF STUDY COHORT ( $\mathrm{N}=433)$

\begin{tabular}{|c|c|c|c|c|c|}
\hline \multirow[b]{2}{*}{ Variables } & \multicolumn{2}{|c|}{ Control $(n=275)$} & \multicolumn{2}{|c|}{ Case $(n=158)$} & \multirow[b]{2}{*}{$\mathrm{P}$ value } \\
\hline & Mean (n) & $\mathrm{SD}(\%)$ & Mean (n) & $\mathrm{SD}(\%)$ & \\
\hline Hemodialysis duration (y) & 9.85 & 4.24 & 14.54 & 5.32 & $<0.001$ \\
\hline Age group & & & & & 0.031 \\
\hline $17-44$ & 29 & 6.95 & 19 & 4.00 & \\
\hline $45-64$ & 164 & 5.20 & 106 & 5.28 & \\
\hline $65-74$ & 60 & 2.93 & 28 & 2.87 & \\
\hline$\geq 75$ & 22 & 4.04 & 5 & 2.79 & \\
\hline Gender & & & & & 0.122 \\
\hline Male & 122 & & 58 & & \\
\hline Female & 153 & & 100 & & \\
\hline Diabetes mellitus & & & & & 0.001 \\
\hline No & 221 & & 144 & & \\
\hline Yes & 54 & & 14 & & \\
\hline \multicolumn{6}{|c|}{ Clinical variables (in first calendar year) } \\
\hline $\mathrm{Hb}(\mathrm{g} / \mathrm{dL})$ & 10.68 & 0.95 & 10.79 & 1.07 & 0.277 \\
\hline Het $(\%)$ & 32.79 & 2.93 & 33.26 & 3.35 & 0.137 \\
\hline Albumin (g/dL) & 3.84 & 0.26 & 3.91 & 0.25 & 0.004 \\
\hline $\mathrm{BUN}(\mathrm{mg} / \mathrm{dL})$ & 69.27 & 13.78 & 72.28 & 12.34 & 0.020 \\
\hline $\mathrm{Cr}(\mathrm{mg} / \mathrm{dL})$ & 10.71 & 2.09 & 11.20 & 2.09 & 0.021 \\
\hline $\mathrm{K}(\mathrm{meq} / \mathrm{L})$ & 4.97 & 0.55 & 5.00 & 0.47 & 0.556 \\
\hline $\mathrm{Ca}(\mathrm{mg} / \mathrm{dL})$ & 9.60 & 0.67 & 9.34 & 0.69 & $<0.001$ \\
\hline $\mathrm{P}(\mathrm{mg} / \mathrm{dL})$ & 5.04 & 0.99 & 5.12 & 1.10 & 0.476 \\
\hline iPTH $(\mathrm{pg} / \mathrm{mL})$ & 458.50 & 394.55 & 245.83 & 325.14 & $<0.001$ \\
\hline Feritin $(\mathrm{ng} / \mathrm{mL})$ & 426.92 & 224.42 & 409.27 & 226.83 & 0.436 \\
\hline $\mathrm{Kt} / \mathrm{V}$ & 1.71 & 0.26 & 1.83 & 0.62 & 0.021 \\
\hline URR & & & & & 0.649 \\
\hline$<.65$ & 5 & 2.25 & 2 & 0.35 & \\
\hline$\geq .65$ & 270 & 5.32 & 156 & 5.39 & \\
\hline Cardiothoracic ratio & & & & & 0.002 \\
\hline$<0.5$ & 107 & 2.80 & 87 & 3.28 & \\
\hline$\geq 0.5$ & 168 & 4.25 & 71 & 3.87 & \\
\hline
\end{tabular}

Control, subjects without parathyroidectomy; Case, subjects with parathyroidectomy.

$\mathrm{iPTH}=$ intact parathyroid hormone; $\mathrm{Ca}=$ corrected serum calcium; $\mathrm{Hct}=$ hematocrit; $\mathrm{Cr}=$ creatinine; $\mathrm{Hb}=$ hemoglobin; $\mathrm{BUN}=$ blood urea nitrogen; $\mathrm{P}=$ phosphorus; $\mathrm{K}=$ potassium; $\mathrm{URR}=$ urea reduction ratio. 


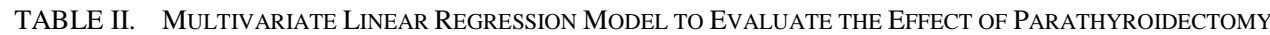

\begin{tabular}{lcccccc}
\hline Dependent variables & Coef. & SE & Pa value & 95 \% CI & R-squared & RMSE \\
iPTH (pg/mL) & 458.502 & 22.387 & $<.001$ & 414.501 to 502.503 & 0.072 & 370.568 \\
$\mathrm{Ca}(\mathrm{mg} / \mathrm{dL})$ & 9.602 & 0.041 & 0 & 9.521 to 9.682 & 0.033 & 0.679 \\
$\mathrm{Hct}(\%)$ & 32.785 & 0.186 & 0 & 32.419 to 33.152 & 0.006 & 3.087 \\
$\mathrm{Cr}(\mathrm{mg} / \mathrm{dL})$ & 10.714 & 0.126 & $<.001$ & 10.466 to 10.962 & 0.012 & 2.090 \\
$\mathrm{Hb}(\mathrm{g} / \mathrm{dL})$ & 10.676 & 0.060 & 0 & 10.558 to 10.794 & 0.003 & 0.996 \\
$\mathrm{BUN}(\mathrm{mg} / \mathrm{dL})$ & 69.269 & 0.801 & $<.001$ & 67.695 to 70.844 & 0.012 & 13.262 \\
$\mathrm{Kt} / \mathrm{V}$ & 1.706 & 0.026 & $<.001$ & 1.656 to 1.659 & 0.018 & 0.426 \\
$\mathrm{Albumin}(\mathrm{g} / \mathrm{dL})$ & 3.836 & 0.016 & 0 & 3.805 to 3.867 & 0.017 & 0.259 \\
$\mathrm{P}(\mathrm{mg} / \mathrm{dL})$ & 5.044 & 0.062 & $<.001$ & 4.921 to 5.166 & 0.001 & 1.029 \\
Ferritin & 426.922 & 13.595 & $<.001$ & 400.201 to 453.644 & 0.001 & 225.040 \\
K (meq/L) & 4.971 & 0.032 & 0 & 4.909 to 5.034 & 0.001 & 0.525 \\
Cardiothoracic ratio & 0.609 & 0.030 & $<.001$ & 0.551 to 0.668 & 0.024 & 0.493 \\
URR & 0.982 & 0.008 & 0 & 0.967 to 0.997 & 0.000 & 0.127 \\
\hline
\end{tabular}

Age, gender, diabetes mellitus, and hemodialysis duration were adjusted in this model.

$\mathrm{P}^{\mathrm{a}}: \mathrm{P}$ value for linear regression coefficient.

$\mathrm{SE}=$ standard error; RMSE $=$ root-mean-square-error .

TABLE III. ACCURACY OF RANDOM FOREST FOR PREDICTING THE EFFECT OF PARATHYROIDECTOMY

\begin{tabular}{|c|c|c|c|c|c|}
\hline \multicolumn{3}{|c|}{ Correctly } & \multicolumn{3}{|c|}{ Positive } \\
\hline & classified & & & predictive & Area under \\
\hline & instances & Sensitivity & Specificity & value & ROC \\
\hline $\begin{array}{c}\text { All patients }(\mathrm{N}= \\
433)\end{array}$ & $76.91 \%$ & $89.1 \%$ & $55.7 \%$ & $76.6 \%$ & $78 \%$ \\
\hline
\end{tabular}

Present study provide further evidence for the benefits of PTX in HD patients. For patients on dialysis, levels of IPTH that are within or below the normal range of the assay area generally indicative of low bone turnover and levels of IPTH that are greater than 2-3 times the upper normal range of the assay are generally indicative of high bone turnover [9]. No change was detected in fracture rates up to 1 year after parathyroidectomy, given that fractures occurred infrequently [10].

Both high calcium and phosphate are implicated in contributing to an increased cardiovascular calcification in patients on renal replacement therapy (RRT) and an increased cardiovascular and all-cause mortality. PTX was associated with improved survival in patients on maintenance dialysis but not in patients with renal allograft. Moreover, PTX improves calcium and phosphate balance [11].

Several studies have found that parathyroidectomy is underutilized [12], and the under treatment seems to hinge on at least 2 factors: first, a lack of diagnostic awareness, and second, deferment of evaluation [13], [14]. The informatics tools reported here can prompt timely referral for further evaluation and treatment. Importantly, this tool utilizes readily available clinical data and provides automated prediction without additional cost or invasive testing. Our Random forest to predict the effect of PTX could become even more accurate as it is "trained" on more patients. Although the present study provided promising evidence for PTX in HD patients, it presents some limitations. First, the study was conducted in just one HD unit. The surgeon's skill, preoperative assessment, and quality of care by nephrologists will affect the outcomes of PTX in HD patients. Second, the determination of test-set accuracies by cross-validation within the dataset, thus making it difficult to predict how the algorithm would perform a different population. Finally, future analysis of the tool should include laboratory values taken at multiple time points to reflect actual clinical practice which may potentially increase the accuracy for the most challenging cases.

\section{Conclusions}

The findings of the present study provide further evidence for the benefits of PTX in HD patients. Incorporation of machine learning (ML) into the healthcare environment can provide opportunities to facilitate and enhance the work of medical experts and ultimately to improve the efficiency and quality of medical care. Moreover, ML can predict the effect of parathyroidectomy on patient survival in secondary hyperthyroidism. 


\section{ACKNOWLEDGMENT}

I would like to express my gratitude to all those who gave me the possibility to complete this paper. The Special thank goes to DIKTI and State University of Padang, Indonesia.

\section{REFERENCES}

[1] Secondary hyperparathyroidism. [Online]. Available: http://www.parathyroid.com.

[2] S. C. Pitt, R. S. Sippel, and H. Chen, "Secondary and tertiary hyperparathyroidism, state of the art surgical management," The Surgical clinics of North America, vol. 5, pp. 1227-1239, 2009.

[3] Parathyroidectomy. [Online]. Available: http://www.medicinenet.net

[4] Introduction to machine learning in healthcare. (March 2017). [Online]. Available: http://www.orionhealth.com/

[5] T. Tanantong, "A KNN approach for ECG signal quality classification," Int. J. on Information and Electronics Eng., vol. 6, pp. 269-272, 2016.

[6] G. D. Magoulas and A. Prentza, "Machine learning in medical applications," ECCAI Advanced Course on Artificial Intelligence for 1999 (ACAI-99), 1999.

[7] J. B. Chen and F. F. Chou, "Association between clinical variables and mortality after parathyroidectomy in maintenance hemodialysis patients," The American Journal of Surgery, vol. 213 pp. 140-145, 2016.

[8] Y. R. Somnay and M. Craven, "Improving diagnostic recognition of primary hyperparathyroidism with machine learning," Surgery, vol. 161 , no. $4,2016$.

[9] A. Ishani and J. Liu, "Clinical outcomes after parathyroidectomy in a nationwide cohort of patients on hemodialysis," Clin. J. Am. Soc. Nephrol., vol. 10, pp. 90-97, January 2015.

[10] Parathyroid Hormone. [Online]. Available: http://www.cari.org.au

[11] K. M. Ivarsson and S. Akaberi, "The effect of parathyroidectomy on patient survival in secondary hyperparathyroidism," Nephrol. Dial. Transplant., vol. 30, no. 12, pp. 2027-2033, September 2015.

[12] B. Wu, P. I. Haigh, and R. Hwang, "Underutilization of parathyrodectomy in enderly patients with primary parathyroidism," J. Clin. Endocrinol. Metab., vol. 95, pp. 43244330, 2010

[13] P. J. Mahadevia, et al., "Clinical management of primary hyperparathyroidism and threshold for surgical referral: A national study examining concordance between practice patterns and consensus panel recommendations," Endocr. Pract., vol. 9, pp. 494-503, 2003.

[14] G. M. London, "Arterial calcification: Cardiovascular function and clinical outcome," Nefrologia, vol. 31, pp. 644-647, October 2011.

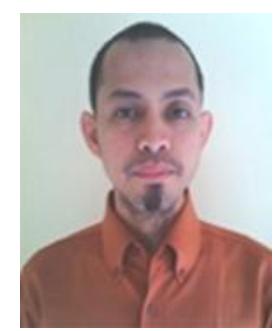

Oktoria is a PhD student of the Department of Electronic Engineering at National Kaohsiung University of Applied Sciences, Taiwan since Fall 2015. He received his Bachelor Degree (S.Pd) from State University of Semarang, Indonesia, 2005. And his Master Degree (M.T) was received from Gadjah Mada University, Indonesia, 2007. Since 2008 he has been being a lecturer at State University of Padang, Indonesia. His research learning and bioinformatics. interests are in genetic algorithm, machine

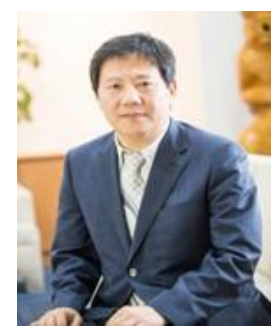

Cheng-Hong Yang is a professor of the Department of Electronic Engineering at National Kaohsiung University of Applied Sciences, Tai-wan. He received his M.S. and $\mathrm{Ph} . \mathrm{D}$. degrees in computer engineering from North Dakota State University in 1988and 1992, respectively.

His principal research interests now are in evolutionary, computation, bioinformatics, and assistive tool implementation.

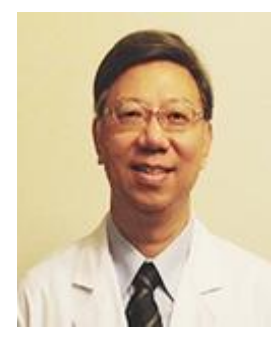

Jin-Bor Chen is a board-certified physician, specializing in hemodialysis, peritoneal dialysis and clinical toxicology. $\mathrm{He}$ is a dynamic member of Taiwan Society of Nephrology serving as a board director and editor of Kidney and Dialysis Journal. He graduated from College of Medicine in Taipei Medical University and continued his residency training at Linkou Chang Gung Memorial Hospital. Thereafter, he was Chang Gung Memorial Hospita promoted to attending physician at Kaohsiung 